\title{
Mutations in autophagy gene cause a rare and severe neurodegenerative disease
}

A new study has shown that de novo mutations in the autophagy gene WDR45 cause static encephalopathy of childhood with neurodegeneration in adulthood (SENDA). "Involvement of autophagy has been suggested in various human neurodegenerative disorders such as Parkinson disease but, until now, mutations in core autophagy genes had not been reported in humans," says Naomichi Matsumoto, who led the study.

SENDA is a rare disease that begins with psychomotor retardation in early childhood, followed by sudden onset of progressive dystonia-parkinsonism and dementia in early adulthood. The sporadic nature of SENDA suggested to Matsumoto and colleagues that de novo or recessive mutations might give rise to the disorder.

Their investigation focused on five patients with SENDA, and began with whole-exome sequencing of the affected individual, an unaffected sibling and, where possible, the unaffected parents.

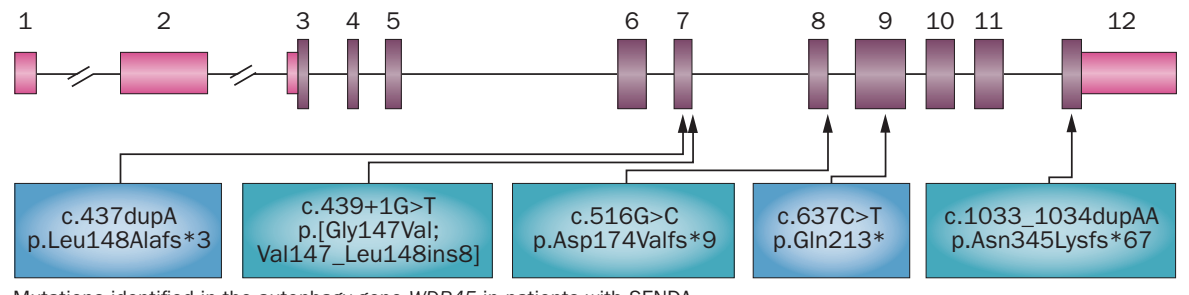

Mutations identified in the autophagy gene WDR45 in patients with SENDA.

"Family-based whole-exome sequencing is extremely powerful for dissecting genetic causes of monogenetic disorders," explains Matsumoto. Each patient was found to harbour a different mutation in WDR45, and the mutations were shown to occur de novo in the three cases in which parental samples were available.

RT-PCR analysis of total RNA extracted from patient-derived lymphoblastoid cell lines (LCLs) reveal that two of the WDR45 mutations were associated with aberrant splicing of the gene transcript, and all five mutations were associated with reduced protein expression. Moroever, an autophagic flux assay revealed impaired autophagic processing in patient-derived LCLs. Lastly, immunofluorescence microscopy indicated aberrant formation of autophagosomes in these cells.

Together, these studies highlight the autophagy pathway as a promising therapeutic target for SENDA.

Katie Kingwell

Original article Saitsu, H. et al. De novo mutations in the autophagy gene WDR45 cause static encephalopathy of childhood with neurodegeneration in adulthood. Nat. Genet. doi:10.1038/ng.2562 